\title{
A Self-Attention Mechanism Neural Network for Detection and Diagnosis of COVID-19 from Chest X- Ray Images
}

\section{Bo Cheng}

Southwest Minzu University

Wei Xiang ( $D$ 21500068@swun.edu.cn )

Southwest Minzu University

Ruhui Xue

Southwest Minzu University

Hang Yang

Southwest Minzu University

Laili Zhu

Southwest Minzu University

\section{Research Article}

Keywords: coronavirus, respiratory diseases, radiologists, medical image

Posted Date: June 14th, 2021

DOI: https://doi.org/10.21203/rs.3.rs-577494/v1

License: (c) (1) This work is licensed under a Creative Commons Attribution 4.0 International License. Read Full License 


\title{
A self-attention mechanism neural network for detection and diagnosis of COVID-19 from chest $x$-ray images
}

\author{
Bo Cheng ${ }^{1}$, Wei Xiang ${ }^{1, *}$, Ruhui Xue ${ }^{1}$, Hang Yang ${ }^{1}$, and Laili Zhu ${ }^{1}$ \\ ${ }^{1}$ Key Laboratory of Electronic and Information Engineering, State Ethnic Affairs Commission, Southwest Minzu \\ University, Chengdu City, Sichuan Province; \\ *corresponding author:21500068@swun.edu.cn
}

\begin{abstract}
The new type of coronavirus is called COVID-19. The virus can cause respiratory diseases, accompanied by cough, fever, difficulty breathing, and in severe cases, it can also cause symptoms such as pneumonia. It began to spread at the end of 2019 and has now spread to all parts of the world. The limited test kits and increasing number of cases encourage us to propose a deep learning model that can help radiologists and clinicians use chest X-rays to detect COVID-19 cases and show the diagnostic features of pneumonia. In this study, our methods are: 1) Propose a data enhancement method to increase the diversity of the data set, thereby improving the generalization performance of the network. 2) Using the deep convolutional neural network model DPN-SE, an attention mechanism is added on the basis of the DPN network, which greatly improves the performance of the network. 3) Use the lime interpretable library to mark the X-ray, the characteristic area on the medical image that is helpful for the doctor to make a diagnosis. The model we proposed can obtain better results with the least amount of data preprocessing given limited data. In general, the proposed method and model can effectively become a very useful tool for clinical practitioners and radiologists.
\end{abstract}

\section{Introduction}

At the end of 2019, a series of new type pneumonia cases was identified, which was caused by the novel coronavirus that was first discovered in 2019.This epidemic was declared constitutes a Public Health Emergency of International Concern in on January 2020 and it was officially named as COVID-19 on February 2020 by WHO ${ }^{1}$. Then COVID-19 began to spread worldwide. Until October 2020, there are 37 million confirmed cases and 1 million people have lost their lives.

Coronaviruses are a group of viruses that cause respiratory tract infections. These viruses are very common in animals, but in some cases it can mutate to infect humans, and then spread rapidly among people. SARS-CoV-2(The virus cause the COVID-19 ${ }^{2}$ ) is the seventh known coronavirus that can infect humans. SARS-CoV-2 can cause respiratory tract infections, Intestinal infections and fewer neurological symptoms. Some infected person of SARS-CoV-2 may faces various sequela after recovery.

After the outbreak of the epidemic, the confirmed cases increased exponentially. As of 6:30 am September 22nd in Beijing time, there are 31,452,367 confirmed cases reported around the world, tragically 968,239 people around the world have lost their lives to this virus and 96 countries confirmed more than 10,000 cases. In the Americas, U.S. Covid-19 death toll approach 200,000. In Africa, the epidemic in Morocco rebounded after loosening control measures.At present, the epidemic is still spreading all over the world, and the risk of sporadic cases and local outbreaks in China still exists. On September 18, 2020, at the Zhongguancun Forum Global Science and life healthforum, Zhong Nanshan, academician of the Chinese Academy of engineering, revealed that the epidemic may still exists in this winter and next spring. It also means that COVID-19 and respiratory diseases such as influenza will come together with the advent of autumn and winter. The task of prevention and control epidemic will be very difficult.

Current evidence suggests that the primary way the virus spreads is by respiratory droplets ${ }^{3,4}$ among people who are in close contact with each other. The incubation period of COVID-19 is generally 1 to 14 days, and the longest is 24 days. In order to effectively prevent and control the epidemic, we not only need to wear masks, wash hands frequently, measure body temperature every day, but also need to trace the source of the virus.

In the early stage of the outbreak, due to the explosive growth of the number of confirmed cases, the demand for suspected case diagnosing also increased, which put great pressure on the medical system with scarce medical resources. The COVID-19 detection reagents currently approved for use mainly include two types, one is nucleic acid detection reagent, and the other is antibody detection reagent. Nucleic acid detection is the main detection method at present, but it takes at least a few hours 
and a maximum of several days, and it has a high professional threshold for operators. And a more fatal drawback is the high probability of false negatives using nucleic acid testing. The advantage of antibody detection is that it takes a short time and the risk to medical staff is lower. However, in the early stage of virus infection, antibody may not be produced in human body, which makes the detection window period exist and there is the possibility of missing detection. Therefore, antibody detection is generally used for auxiliary detection of negative cases that test by nucleic acid or rapidly screening potential cases in the population, but it cannot replace nucleic acid detection.

The limitations of detection methods and the current global epidemic situation encourage us to try to propose a deep network learning model ${ }^{4-6}$. We use self-attention mechanism and advanced image classification algorithm to intelligently diagnose and quantitatively evaluate X-ray images, and marked the severity of various pneumonia diseases including local lesions, diffuse lesions and whole lung involvement, which can help doctors identify lesions more quickly and accurately. The model focus on the lesion site, doctors can directly focus on the labeled part and make diagnosis quickly, which greatly shortens the diagnosis time of COVID-19.

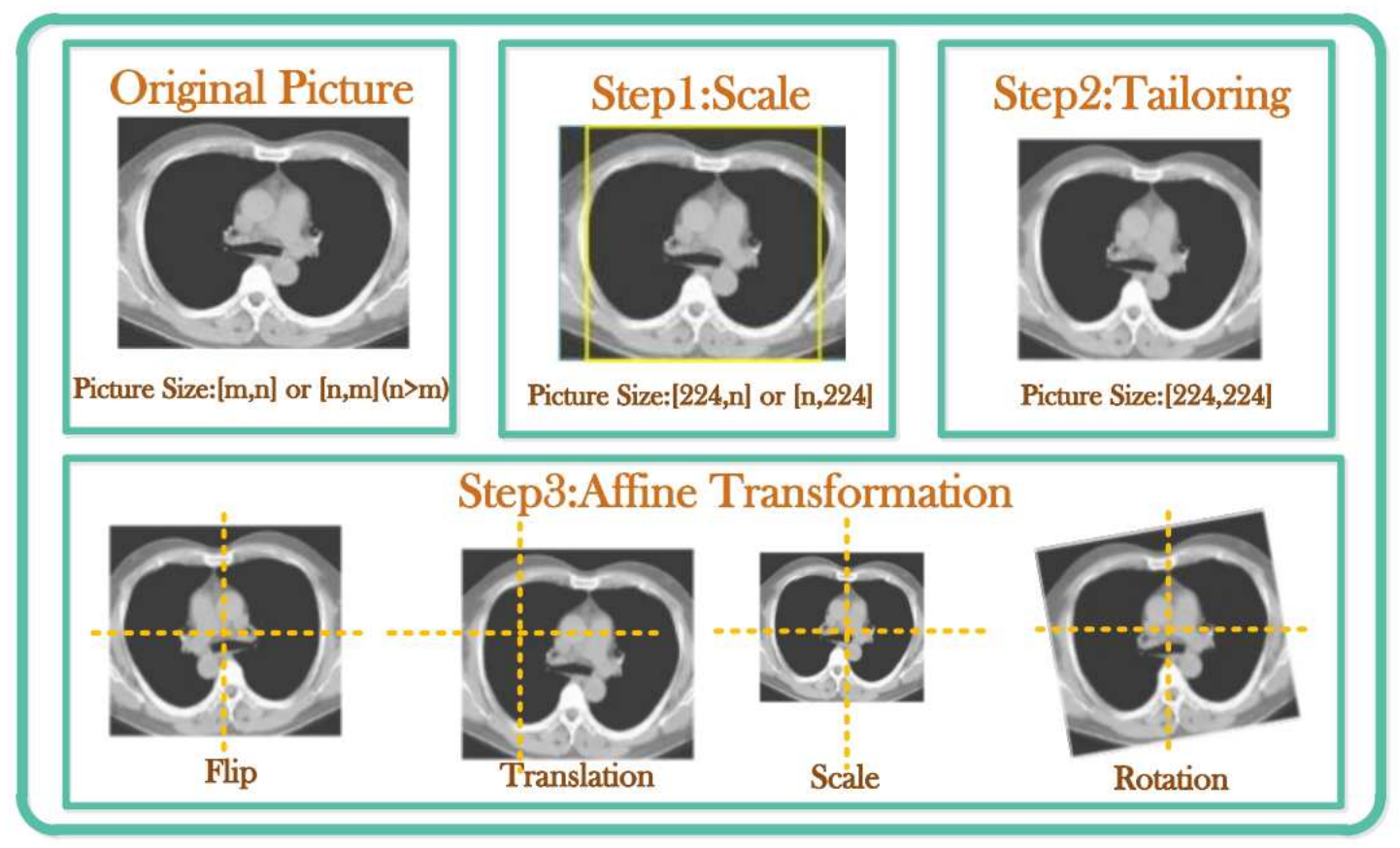

Figure 1. Overview of the Data Augmentation. Step1: Scale the narrow side of the image to size 224; Step2: Randomly tailoring a picture of size [224, 224] from an image of [224, n] or [n,224]; Setp3: The [224, 224] size picture is processed by affine transformation: flip, rotate, scale.

\section{Related Work}

With the rise of artificial intelligence, deep learning has been gradually used in health-related fields, and its wide use in other fields has proved that deep learning can help people solve some basic problems. In this chapter, we review and summarize the literature on the application of artificial intelligence technology to detect chest diseases

With the global outbreak of covid-19, people in different fields have made different contributions to against this epidemic. The pioneers of artificial intelligence through the technology of image classification to classify chest CT and X-ray images ${ }^{7-12}$ - Each other put forward different deep learning network architecture to diagnose patients. With the rapid development of artificial intelligence, computer classification of images has been proven to have a higher accuracy rate than human eye recognition ${ }^{13}$ (many network models have better classification effects on ImageNet data sets than ordinary people's judgment effects). Many researchers are committed to improving the detection and analysis methods of various diseases by acquiring radiology data sets and applying data science classifiers

Hemdan ${ }^{14}$ used a deep learning model to diagnose COVID-19 in X-ray images and proposed a COVIDX-Net model with 7 convolutional layers. Wang and Wong ${ }^{15}$ proposed a deep model (COVID-Net) for COVID19 detection, which achieved $92.4 \%$ accuracy when classifying normal, non-COVID pneumonia and COVID-19. Ioannis developed a deep learning model using 
224 COVID-19 images ${ }^{16}$. Their model achieved accuracy rates of $98.75 \%$ and $93.48 \%$ in 2 and 3 categories, respectively. Narin used chest X-ray images coupled with the ResNet50 model to obtain 98\% COVID-19 detection accuracy ${ }^{17}$. Sethy and Behera used a support vector machine (SVM) classifier to classify features obtained from various convolutional neural network $(\mathrm{CNN})$ models using X-ray images ${ }^{18}$. It shows that the ResNet50 model with SVM classifier has the best performance. Finally, there are some recent studies on COVID-19 detection. These studies use various deep learning models with CT images ${ }^{19-24}$.

Li designed the COVNet neural network for COVID-19 detection to extract visual features from CT images ${ }^{25}$. Their model uses a RestNet50 network with input CT images. They performed 4,356 chest CT scans on 3,322 patients (1296 CT scans for COVID-19, 1,735 CAP and 1,325 non-pneumonia), with an accuracy equal of 0.96 .

Fan et al. proposed an Inf-Net ${ }^{26}$ segmentation model to automatically identify infected areas from CT slices. In Inf-Net, a parallel partial decoder is used to aggregate the high-level features and generate a global map. Then, the implicit reverse attention and explicit edge-attention are utilized to model the boundaries and enhance the representations. The first model is a network based on ResNet18, then the second model is designed on the first network to construct a structure by connecting the location attention mechanism in the fully connected layer, with the purpose of improving the overall accuracy. Their dataset consists of 1,710 CT images, including 357 COVID-19, 390 influenza A viral pneumonia, and 963 normal.

\section{Method}

In this section, we will discuss the data processing methods, the implementation of model architecture and training methods.

\section{Data Augmentation}

Because it is difficult to obtain chest X-ray images of patients with new coronary pneumonia,we have collected fewer chest X-ray images of patients with new coronary pneumonia (the training set contains 1119 images, and the test set contains 293 images,for a total of 1412 images). Using a small number of data to train the network model can easily cause the model to overfit, resulting in very low recognition accuracy on the test set. In the field of computer vision, image enhancement is a common implicit regularization technique to reduce overfitting in deep convolutional neural networks, and is widely used to improve the performance of benchmark datasets. ${ }^{27,28}$.Common image enhancement methods include certain variations and combinations of flipping, rotating, scaling, and cropping. Different fields, imaging methods and tasks may benefit from a variety of image transformations and combinations ${ }^{27}$. For example, in the medical image analysis done in this article, compared with natural images, the data set is usually small and difficult to obtain, and the details in the image are very important, which may be the basis for doctors to distinguish diseases. So when we do data enhancement, we should not modify the original image too much, otherwise the details on the x-ray image will be eliminated. After many attempts in the experiments of this paper, it is found that the data enhancement process shown in Fig. 1 can achieve better results. The whole process goes through three steps, step1: Scale the narrow side of the image to size 224; setp2: Randomly tailoring a picture of size $[224,224]$ from an image of $[224, n]$ or $[\mathrm{n}, 224]$ size; setp3: The $[224,224]$ size picture is processed by affine transformation: flip, rotate, scale.

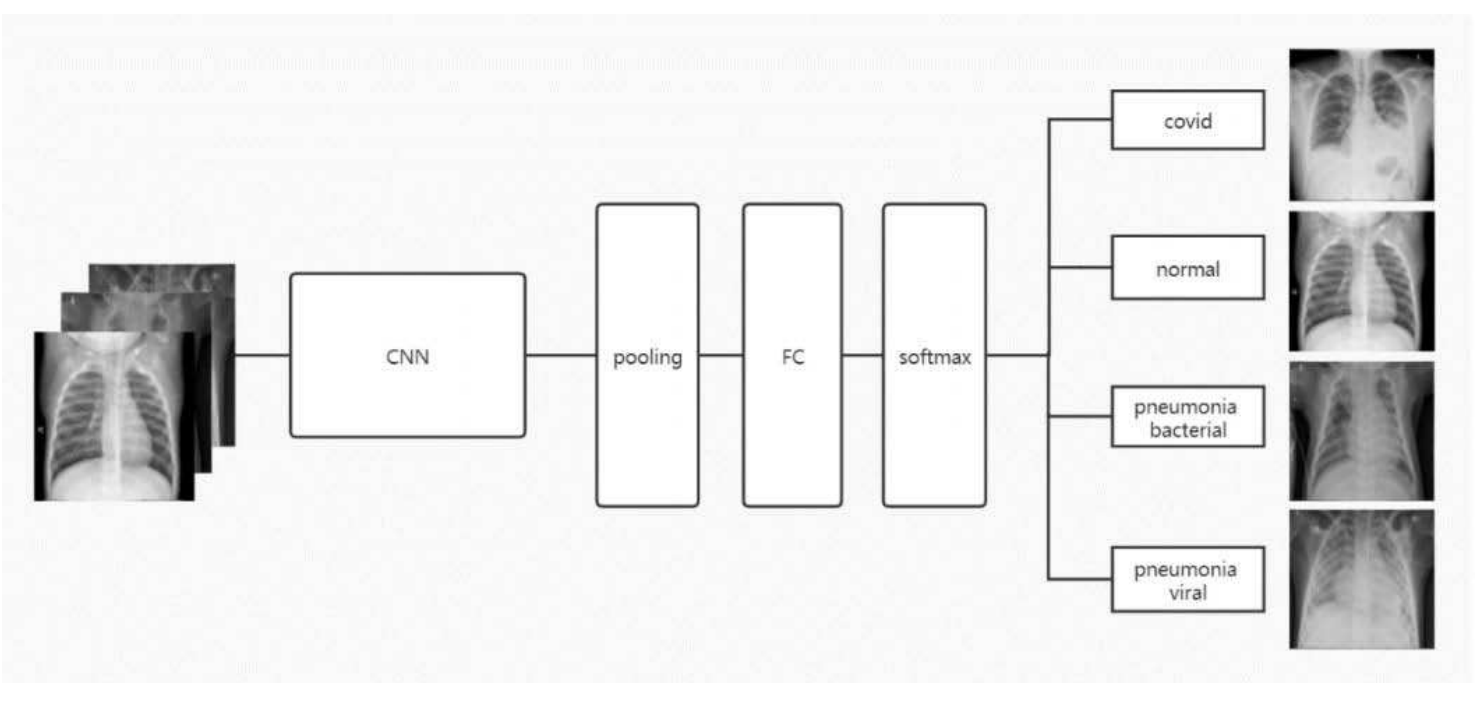

Figure 2. Network overall framework diagram 


\section{Network Model}

In this section, we will discuss the whole network framework, which is based on the convolutional neural network in deep learning to classify covid19 x-ray images. The overall framework is shown in Fig. 2. The main framework of the network is the DPNNet ${ }^{29}$, and the structure is shown in Figure 1. This network combines the advantages of ResNet ${ }^{30}$ and DenseNet ${ }^{31}$, and won the championship in the 2017 ImageNet classification competition ${ }^{32}$. In this paper, we use a 92-layer DPN network, which started with a $7 * 7$ convolutional layer and maximum pooling layer. Then there are 4 stages, each stage is composed of multiple substages. The core idea of substage is to integrate ResNet and DenseNet. ResNet: focuses on the reuse of features and the sharing of parameters; DenseNet: focuses on the discovery of new features and Achieve complementary advantages and disadvantages. Followed by an average pooling layer and a fully connected layer, and finally a softmax layer. However, because DPN-92 is composed of a large number of convolutional layers, and the convolution kernel, as the core of the convolutional neural network, generally can only obtain feature maps from the local receptive field, which lacks the characteristics of the global receptive field. So we introduced the attention mechanism network on the basis of DPN-92.

The human attention visual mechanism uses limited attention to quickly screen high-value information from a large amount of information. The attention mechanism in deep learning is essentially similar to the selective visual attention mechanism of humans. Its core goal is to select information that is more critical to the current task goal from a large number of information. At present, the attention mechanism network has been widely used in various deep learning applications such as natural language processing, image recognition, and speech recognition. In this article, we use the senet attention mechanism network.

Senet is mainly composed of three parts: compression part, excitation part and reweight part ${ }^{33}$. The compression part compresses the features along the spatial dimension, turning each two-dimensional feature channel into a real number. This real number has a global receptive field to some extent, and matches the dimension of the input and the number of feature channels of the input. It represents the global distribution of the response on the feature channel. This part is implemented using global pooling.

The incentive part is similar to the gate mechanism in a recurrent neural network ${ }^{4}$. A weight is generated for each feature channel through parameters, and a correlation between feature channels is modeled by learning parameters. The last part is reweight, and we regard the weight of the output of the excitation part as the weight of each feature after feature selection The degree of importance is then weighted channel by channel by multiplication to the first input feature to complete the recalibration of the input feature in the channel dimension. The senet network is introduced into the DPN- 92 network, and the network part becomes the DPN- SE network, as shown in Fig. 3.
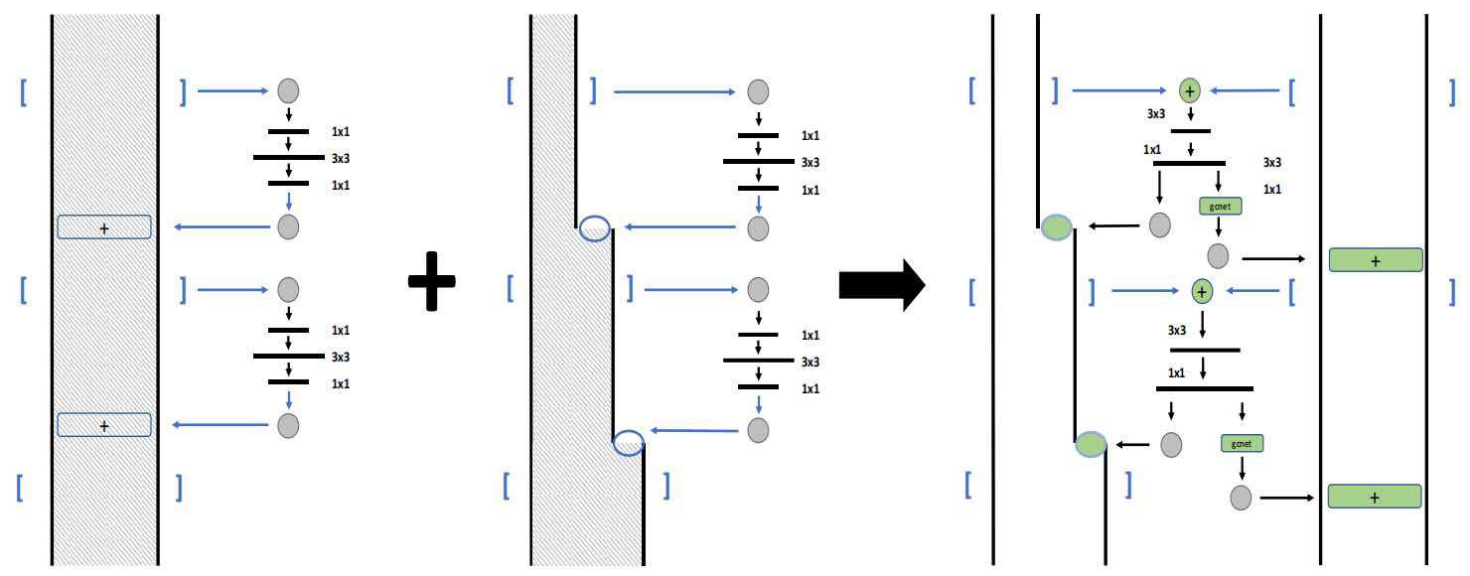

Figure 3. Network overall framework diagram

\section{Model Explanation}

With the rapid development of artificial intelligence, artificial intelligence has also had a profound impact on all walks of life. From Meitu software to facial recognition in train stations, systems built around artificial intelligence have had a huge impact on medical care, transportation, criminal justice, financial risk management, and other areas of society. Although artificial intelligence is widely used, machine learning models are still black boxes. Although these models show capabilities beyond humans, we are not yet sure what specific information in the input data will make them make decisions. Deep learning models usually contain deeply nested non-linear structures, making them less transparent. The lack of transparency is not a problem 
for AlphaGo itself. However, in areas where interpretability and transparency are critical (such as medical diagnosis, military and combat operations), the opacity of the model greatly hinders the expansion of AI/ML.

From a broad perspective, interpretability ${ }^{34,35}$ means that when we need to solve a thing or make a decision about it, we need to obtain information from this thing that can be understood enough to help us make a decision. Therefore, in this article, we need to clearly mark the basis for the model judgment on the chest X-ray image, so that the doctor can quickly find the lesion area and judge whether the marked part is the basis for judgment.

In the study of local interpretability of machine learning models, a representative method is Local Interpretable ModelAgnostic Explanation (LIME) ${ }^{36}$ proposed by Marco Tulio Ribeiro et al. The main idea of LIME is to use interpretability models (such as linear models, decision trees) to locally approximate the prediction of the target black box model. This method does not go deep into the model. By slightly perturbing the input, detecting changes in the output of the black box model, and training an interpretable model at points of interest (original input) based on this change. It is worth noting that the interpretability model is a local approximation of the black box model, rather than a global approximation, which is the origin of its name. The mathematical expression of LIME is as follows:

$$
\operatorname{explanation}(x)=\underset{g \varepsilon G}{\operatorname{argmin}} L(f, g, \pi x)+\Omega(g)
$$

For the interpretation model $g$, we compare the approximation between $g$ and the original model $f$ by minimizing the loss function, where $\Omega(g)$ represents the model complexity of the explanation model $g$,and $G$ represents all possible explanation models(If we use linear models to explain, $G$ means all linear models), $\pi x$ defines the realm of $x$. We make the model interpretable by minimizing $L$. Among them, the domain size and complexity of the model need to be defined.

Below is a brief description of LIME's workflow. In order to be independent of the model, LIME will not go deep into the model. In order to find out which part of the input contributes to the prediction result, we will make a slight disturbance around the input value and observe the prediction behavior of the model. Then we assign weights based on the distance of these disturbed data points from the original data, and learn an interpretable model and prediction results based on them. The demonstration diagram of this process is as follows. The decision function of the original model is represented by a blue/pink background, which is obviously non-linear. The bright red cross indicates the sample being interpreted (called X). We sample around $\mathrm{X}$ and assign weights according to their distance to $\mathrm{X}$ (where weight means size) We use the original model to predict these disturbed samples, and then learn a linear model (dotted line) to approximate the model well near X. Note that this explanation only holds near $\mathrm{X}$ and is invalid for the whole world.

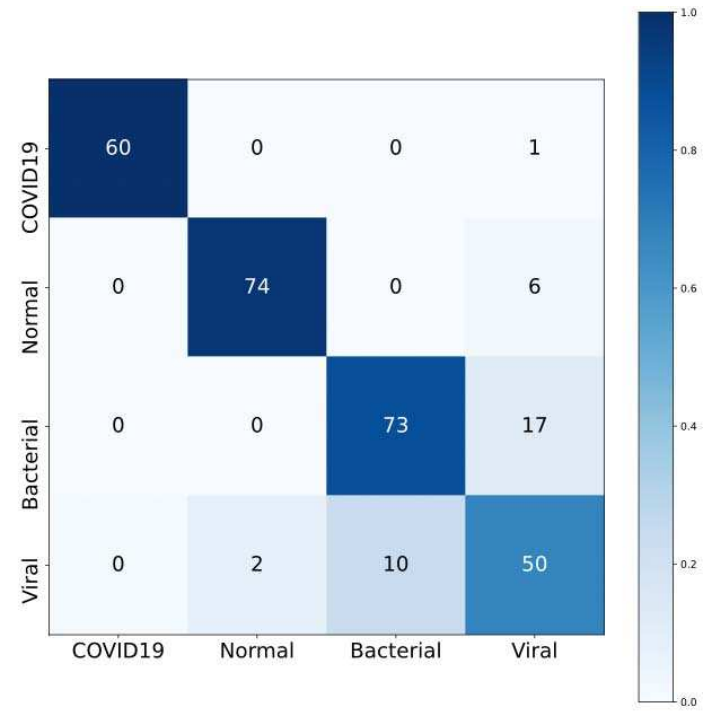

(a)

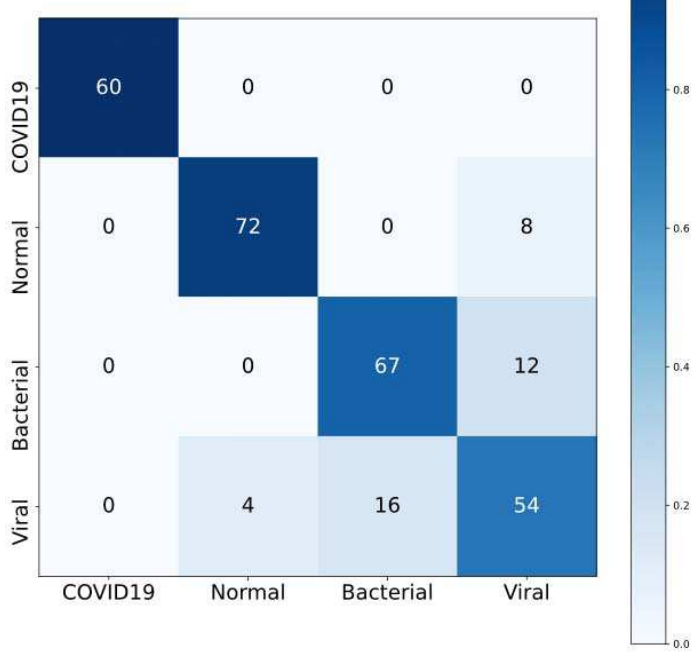

(b)

Figure 4. Schematic diagram of a four-level tripod-type atomic system driven by three coherent laser fields. 


\section{Experiment}

In this section, we will introduce the basic information of the data set and analyze the experimental data results according to the following aspects:

- Detect the effects of using data enhancement and unused data enhancement;

- Use the DPN-SE network model to test the recognition accuracy;

- Use an interpretable model to highlight the key areas of the X-ray picture of the new crown.

\section{Dataset}

The accuracy of model training largely depends on the data set. COVID-19 is a new disease. We need to select a large number of chest X-rays to allow our model to fully learn the characteristics of the lungs. The X-ray images of COVID-19 are available on GitHub by Joseph et al. ${ }^{37}$.

The author collected chest X-ray images of new coronary pneumonia from various real sources of the Radiological Society of North America. Our data set is a four-category data set, which not only contains chest X-ray radiographic images of new coronary pneumonia, but also contains bacterial pneumonia, viral pneumonia and normal pneumonia from the Kaggle repository "Chest X-Ray Images (Pneumonia) ${ }^{38}$ Chest X-ray image. This data set consists of 1203 normal chest radiographs, 660 bacterial pneumonia chest radiographs and 931 viral pneumonia chest radiographs. The data set for our model training comes from the data set compiled by ${ }^{39}$. Among them, the new crown Pneumonia pictures: 304 pictures, normal pictures: 375 pictures, bacterial pneumonia: 379 pictures, viral pneumonia: 354 pictures. In order to avoid over-fitting problems in the later stage, we used data enhancement technology. The four categories of pictures are shown in Fig. 5.

\section{Results and Discussion}

In this study, the chest X-ray dataset mentioned in Section 4.1 was used to train the model. The network structure we use is VGG16, ResNet, InceptionV4, DenseNet, DPNNet and our own DPN-SE network with channel self-attention mechanism added. First, compare the trained network after data enhancement with the directly trained network (the method of data enhancement is introduced in section 3.1). In the experiment, all the network models were trained for 100 epochs, and the cost loss graph was observed, and finally reached the convergence state. The four performance indicators for evaluating the classification model are:

$$
\begin{gathered}
\text { Accuracy }=\frac{T N+T P}{T N+T P+F N+F P} \\
\text { Recall }=\frac{T P}{T P+F N} \\
\text { Precision }=\frac{T P}{T P+F P} \\
F 1-\text { Score }=2 \times \frac{\text { Precision } \times \text { Recall }}{\text { Precision }+ \text { Recall }}
\end{gathered}
$$

TN, TP, TN, and FN in formulas (1)-(4) represent the number of true, false positive, true negative, and false negative, respectively. TP is the proportion of model prediction results that are correctly marked as positive; FP is the proportion of incorrectly marked as positive; TN is the proportion of correctly marked as false, and FN is the proportion of incorrectly marked as false (COVID- 19 is the positive category, and the other categories are the negative category).

In Fig. 4., we present the recognition results trained by the DPN131 network structure in the form of a confusion matrix $(\mathrm{CM})$, a) the image is the recognition effect without image enhancement, and b) the image is the recognition effect with image enhancement. In the comparison of confusion matrix a) and b), we can observe that after adding data enhancement, the average accuracy rate has risen from 0.8089 to 0.8328 . The accuracy rate for the COVID-19 category rose from 0.92 to 0.97 , the recall rate rose from 97 to 98 , and the F1-score rose from $94 \%$ to $98 \%$. Several other categories of indicators have also increased. In order to add more verification examples, we used other models for comparison. As shown in Table.1, a variety of different network models are used to test the effects of data enhancement and unused data enhancement. The evaluation indicators include accuracy, precision, recall, $\mathrm{F}$ value The calculation of the indicator counts the COVID-19 category as a positive example, and the others as a negative example).

As can be seen from the data in Table. 1, we have used 10 network models such as ResNet, DenseNet, DPNNet, VGG16, Inceptionv4, etc. to test. The recognition accuracy of most models is above $80 \%$. Comparison of network models using ResNet50. If the data set without data enhancement is used to train the network, the average accuracy obtained is $79 \%$, the precision is 0.92 , the recall rate is 0.98 , and the F-measure is 0.95 . If the network is trained on the data set processed by 


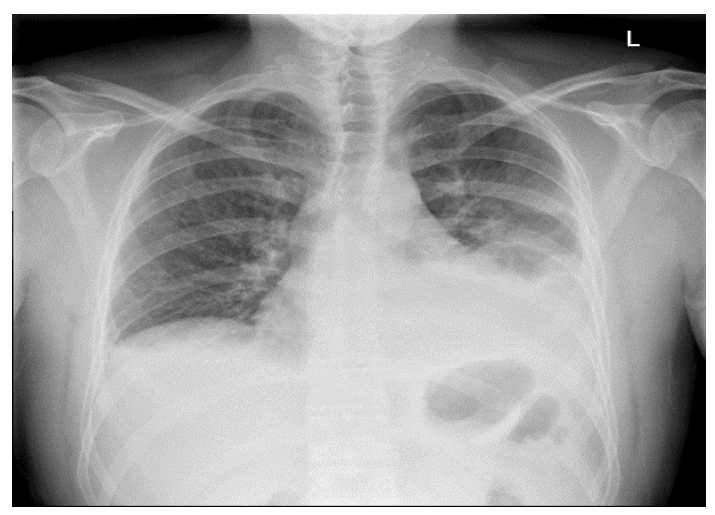

(a) Covid-19

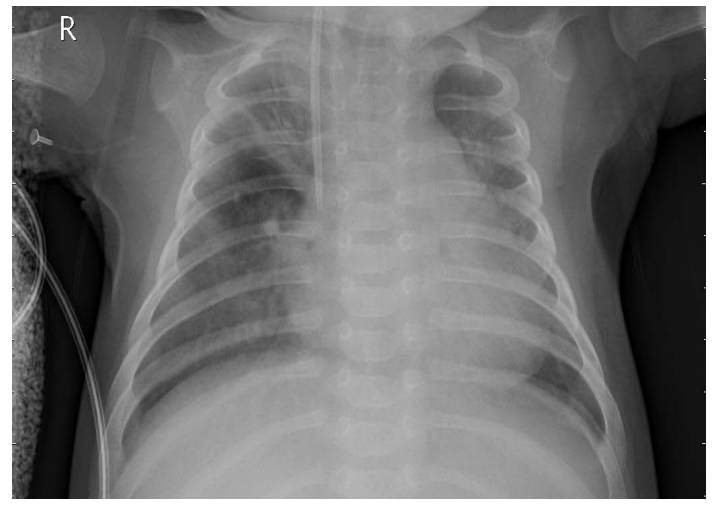

(c) Pneumonia Bacterial

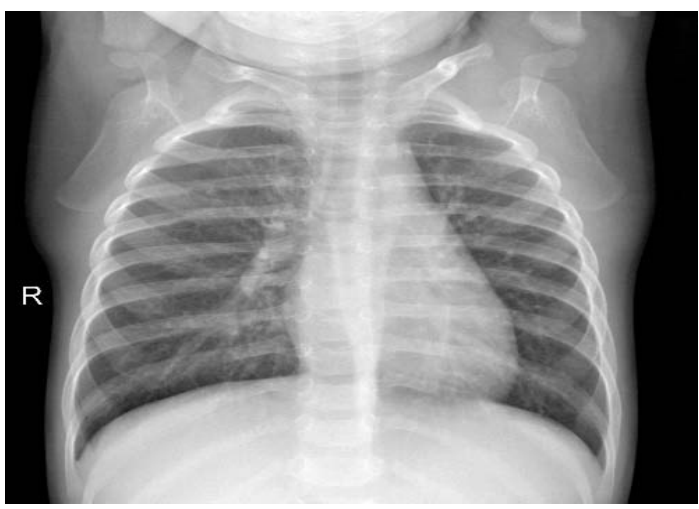

(b) Normal

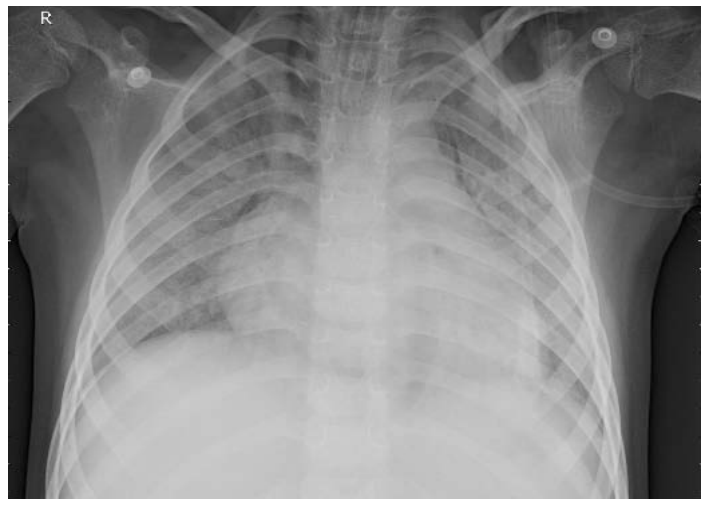

(d) Pneumonia Viral

Figure 5. Types of lung X-ray images

data enhancement, the average accuracy obtained is $80 \%$, the precision is 0.97 , the recall rate is 0.98 , and the F-measure is 0.98. It can be seen from the comparison that after data enhancement processing, the trained network model can improve the recognition accuracy by $1 \%$. Among the 10 network models displayed in total, the recognition effect of 7 network models has improved after data enhancement processing, the recognition effect of 2 network models has decreased, and the recognition effect of 1 network model has basically remained unchanged. Finally, we can infer that after processing the x-ray sample data using data enhancement methods, training the network model can effectively improve the recognition accuracy of about $1 \%$.

\begin{tabular}{|c|c|c|c|c|c|c|c|c|}
\hline \multirow{2}{*}{ Model } & \multicolumn{4}{|c|}{ Data Enhancement } & \multicolumn{4}{c|}{ Without Data Enhancement } \\
\cline { 2 - 9 } & Accuracy & Precision & Recall & F1-score & Accuracy & Precision & Recall & F1-score \\
\hline Resnet18 & 0.77 & 0.96 & 0.80 & 0.87 & 0.78 & 0.90 & 0.93 & 0.92 \\
\hline Resnet50 & 0.79 & 0.92 & 0.98 & 0.95 & 0.80 & 0.97 & 0.98 & 0.98 \\
\hline Resnet101 & 0.81 & 0.94 & 0.97 & 0.95 & 0.78 & 0.98 & 0.90 & 0.94 \\
\hline DenseNet121 & 0.82 & 0.98 & 0.98 & 0.98 & 0.84 & 0.97 & 0.98 & 0.98 \\
\hline DenseNet201 & 0.87 & 0.98 & 1.00 & 0.99 & 0.86 & 1.00 & 1.00 & 1.00 \\
\hline DPN68 & 0.74 & 0.97 & 0.95 & 0.96 & 0.81 & 0.93 & 0.95 & 0.94 \\
\hline DPN92 & 0.80 & 0.89 & 0.98 & 0.94 & 0.80 & 0.94 & 1.00 & 0.97 \\
\hline DPN131 & 0.80 & 0.92 & 0.97 & 0.94 & 0.83 & 0.97 & 0.98 & 0.98 \\
\hline VGG16 & 0.79 & 0.91 & 0.95 & 0.93 & 0.81 & 0.93 & 0.95 & 0.94 \\
\hline InceptionV3 & 0.82 & 0.94 & 0.97 & 0.95 & 0.82 & 0.94 & 0.97 & 0.95 \\
\hline
\end{tabular}

Table 1. Use a variety of different network models to test the effects of data enhancement and unused data enhancement. The evaluation indicators include accuracy, precision, recall, F value (precision rate, recall rate, $\mathrm{F}$ value and other indicators). -19 categories are regarded as positive examples, others are false examples)

Our purpose is to improve the recognition accuracy of the network. In addition to using data enhancement to increase the recognition accuracy, we also want to modify the network model to increase the recognition accuracy. The main framework 


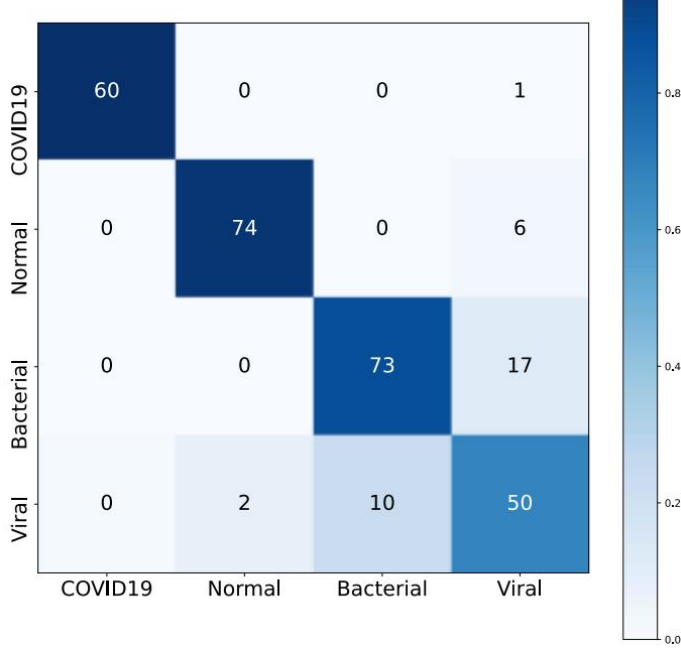

(a)

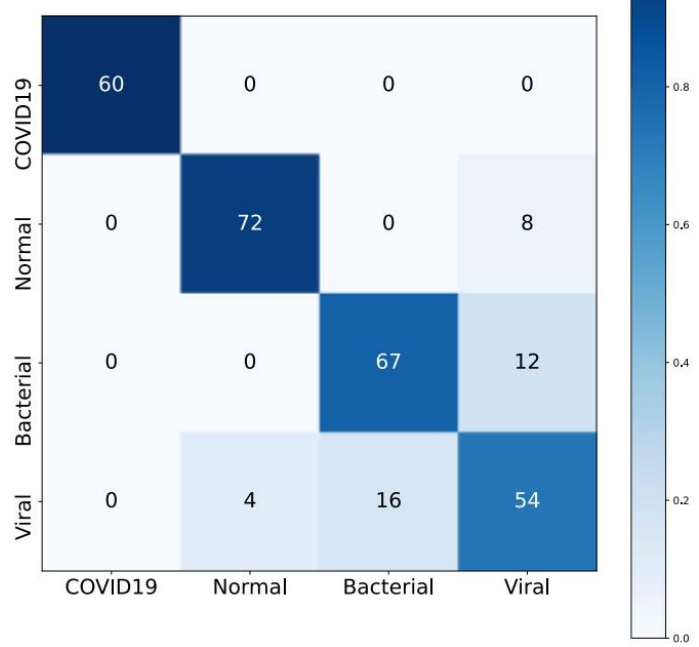

(b)

Figure 6. (a) the results of DPNNet network model training (b) the results of DPNN-SE network model training.

of the network is DPN network [29]. We use a DPN-92 network, which starts with a $7 * 7$ convolution layer and a maximum pooling layer, and then there are four stages, each stage is composed of multiple substages. On the basis of DPN-92, attention mechanism network is introduced. The data set during training is processed by the data enhancement described in Section 3.1

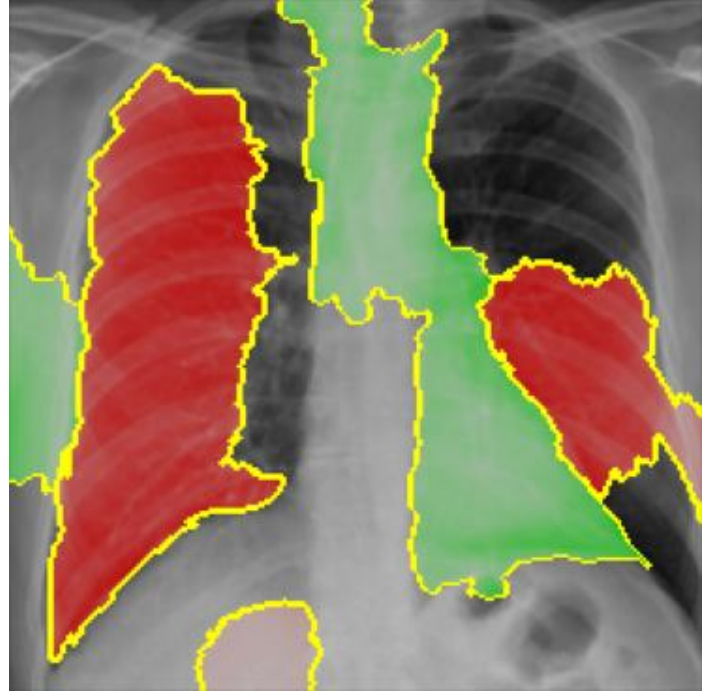

(a)

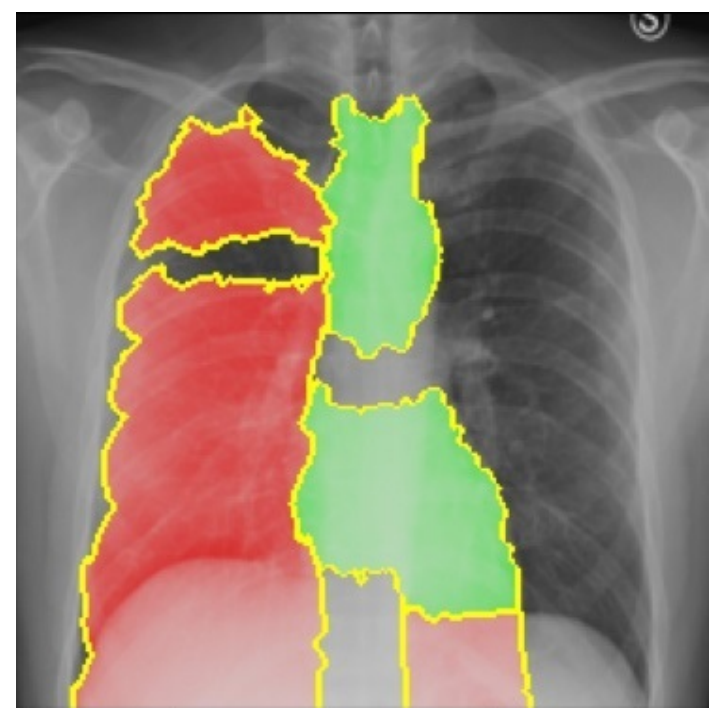

(b)

Figure 7. Examples of COVID-19 model activation maps.

As shown in Fig. 6, we show the confusion matrix of the recognition effect of the classic DPN network and the modified DPN-SE network with the self-attention mechanism added. Table 2 shows the accuracy, precision, recall rate and F-measure of DPN on the test set. Table 3 shows the accuracy, precision, recall rate and F-measure of DPN-SE on the test set. In the confusion matrix, it can be seen that this test set has 64 cases of new coronary pneumonia, 89 normal cases, 76 cases of viral pneumonia, and 64 cases of viral pneumonia. The classification performance of viral pneumonia and bacterial pneumonia is lower than that of the other two categories, resulting in a lower overall accuracy rate. If we combine bacterial pneumonia and viral pneumonia into pneumonia, the overall accuracy will be significantly improved. The good news is that our detection of 


\begin{tabular}{|c|c|c|c|}
\hline Class & Precision & Recall & F-measure \\
\hline COVID-19 & 0.98 & 0.98 & 0.98 \\
\hline Normal & 0.92 & 0.95 & 0.94 \\
\hline Pneumonia Bacterial & 0.1 & 0.87 & 0.78 \\
\hline Pneumonia Viral & 0.72 & 0.51 & 0.60 \\
\hline Overall accuracy & \multicolumn{3}{|c}{0.82} \\
\hline
\end{tabular}

Table 2. Performance of DPNNet

\begin{tabular}{|c|c|c|c|}
\hline Class & Precision & Recall & F-measure \\
\hline COVID-19 & 0.97 & 0.98 & 0.98 \\
\hline Normal & .94 & 0.96 & 0.95 \\
\hline Pneumonia Bacterial & 0.1 & 0.87 & 0.78 \\
\hline Pneumonia Viral & 0.74 & 0.88 & 0.81 \\
\hline Overall accuracy & \multicolumn{3}{|c}{0.84} \\
\hline
\end{tabular}

Table 3. Performance of DPN-SE

new crowns and normal lungs has a high accuracy rate. The average accuracy rate on the DPN network is $82 \%$, the precision is $98 \%$, the recall rate is $98 \%$, and the F-measure is $98 \%$. On the DPN-SE network, the average accuracy rate is $84 \%$, the precision is $97 \%$, the recall rate is $98 \%$, and the F-measure is $98 \%$. The average accuracy rate has improved by $2 \%$. The positive results obtained in the experimental data are about the high accuracy and recall rate of the COVID-19 category. A higher recall rate means a lower false negative (FN), and a lower number of false negatives (FN) is the result we hope to get. This is very important because minimizing missed cases of COVID-19 is an important goal of medical diagnosis. In general, when the attention mechanism structure is added to the network model, it can be observed that the average accuracy of the DPN-SE network has increased by $2 \%$, indicating that the modified network model has played a role.

We need to clearly mark the features judged by the model on the chest X-ray image, so that the doctor can quickly find the lesion area and judge whether the marked part is the basis for judgment. Fig. 7 shows an example of activation diagram that can explain model processing using lime. This method does not go deep into the model. It detects changes in the output of the black box model by slightly perturbing the input, and trains an interpretable model at the point of interest (original input) based on this change. Only x-ray images and the model that has been trained can get the activation map. The decision of the Lime model is represented by a red/blue background, in which the red area represents the key feature that the classification model focuses on, and the blue area is the area of unnecessary attention. It can be observed from the figure that the red key feature areas are basically distributed inside the chest cavity, which requires special attention. The blue area is the non-pulmonary area in the middle and edge of the body.

The environment we used for the experiment was 1080TiGPU, and it took $3 \mathrm{~s}$ to analyze the category of a picture and visualize the activation map on a single GPU.

\section{Conclusion}

With the COVID-19 pandemic, the number of cases is increasing. Many places are facing the challenge of shortage of testing resources. In this article, we propose a deep neural network model DPN-SE that uses chest X-rays to identify COVID-19 cases. And for a small number of samples, data enhancement methods are used, and good results have been achieved on the test set. Compared with DPN-SE, DPN-SE has the same computational overhead as the DPN network structure, but it improves the recognition accuracy by about $2 \%$. When more training samples are used, the performance can be further improved. The accuracy of the recognition results of our model is very high, which is believed to help radiologists have a deeper understanding of case related to COVID-19.

\section{Source code and dataset}

In order for everyone to continue the research well, we provide the code and data set for the experimental research. The trained model and data can be obtained here:

https : //github.com/ChengBo5/covid19-X-ray.git 


\section{Acknowledgments}

This work is financially supported by the Fundamental Research Funds for Central University, Southwest Minzu University ( 2020NYBPY02).

\section{References}

1. Wu, C. et al. Risk factors associated with acute respiratory distress syndrome and death in patients with coronavirus disease 2019 pneumonia in wuhan, china. JAMA internal medicine 180, 934-943 (2020).

2. Yi, C. et al. Key residues of the receptor binding motif in the spike protein of sars-cov-2 that interact with ace 2 and neutralizing antibodies. 17, 621-630, DOI: 10.1038/s41423-020-0458-z (2020).

3. Hall, E. et al. Nowcasting for real-time covid-19 tracking in new york city: An evaluation using reportable disease data from early in the pandemic. JMIR Public Heal. Surveillance 7, e25538, DOI: 10.2196/25538 (2021).

4. Fanelli, D. \& Piazza, F. Analysis and forecast of covid-19 spreading in china, italy and france. Chaos, Solitons \& Fractals 134, 109761, DOI: https://doi.org/10.1016/j.chaos.2020.109761 (2020).

5. Wang, X. et al. A weakly-supervised framework for COVID-19 classification and lesion localization from chest CT. IEEE Transactions on Med. Imaging 39, 2615-2625, DOI: 10.1109/tmi.2020.2995965 (2020).

6. Gozes, O. et al. Rapid ai development cycle for the coronavirus (covid-19) pandemic: Initial results for automated detection \& patient monitoring using deep learning ct image analysis. (2020). 2003.05037.

7. Hall, L. O., Paul, R., Goldgof, D. B. \& Goldgof, G. M. Finding covid-19 from chest x-rays using deep learning on a small dataset (2020). 2004.02060.

8. Alimadadi, A. et al. Artificial intelligence and machine learning to fight covid-19. Physiol. Genomics 52, 200-202, DOI: 10.1152/physiolgenomics.00029.2020 (2020). PMID: 32216577, https://doi.org/10.1152/physiolgenomics.00029.2020.

9. Chen, X. et al. A diagnostic model for coronavirus disease 2019 (COVID-19) based on radiological semantic and clinical features: a multi-center study. Eur. Radiol. 30, 4893-4902, DOI: 10.1007/s00330-020-06829-2 (2020).

10. Gore, J. C. Artificial intelligence in medical imaging. Magn. Reson. Imaging 68, A1-A4, DOI: 10.1016/j.mri.2019.12.006 (2020).

11. Hemdan, E. E.-D., Shouman, M. A. \& Karar, M. E. Covidx-net: A framework of deep learning classifiers to diagnose covid-19 in X-ray images (2020). 2003.11055.

12. Mor, N. S. Corona virus disease (covid-19) screening with deep learning using ct images. (2020).

13. Apostolopoulos, I. D. \& Mpesiana, T. A. Covid-19: automatic detection from x-ray images utilizing transfer learning with convolutional neural networks. Phys. Eng. Sci. Medicine 43, 635640, DOI: 10.1007/s13246-020-00865-4 (2020).

14. Narin, A., Kaya, C. \& Pamuk, Z. Automatic detection of coronavirus disease (covid-19) using X-ray images and deep convolutional neural networks. Pattern Analysis Appl. DOI: 10.1007/s10044-021-00984-y (2021).

15. Wang, L., Lin, Z. Q. \& Wong, A. COVID-net: a tailored deep convolutional neural network design for detection of COVID-19 cases from chest x-ray images. Sci. Reports 10, DOI: 10.1038/s41598-020-76550-z (2020).

16. Song, Y. et al. Deep learning enables accurate diagnosis of novel coronavirus (COVID-19) with CT images. IEEE/ACM Transactions on Comput. Biol. Bioinforma. 1-1, DOI: 10.1109/tcbb.2021.3065361 (2021).

17. Wang, S. et al. A deep learning algorithm using CT images to screen for corona virus disease (COVID-19). Eur. Radiol. DOI: 10.1007/s00330-021-07715-1 (2021).

18. Sethy, P. K. \& Behera, S. K. Detection of coronavirus disease (COVID-19) based on deep features. DOI: 10.20944/ preprints202003.0300.v1 (2020).

19. Zhao, W., Zhong, Z., Xie, X., Yu, Q. \& Liu, J. Ct scans of patients with 2019 novel coronavirus (covid-19) pneumonia. Theranostics 10, 4606-4613, DOI: 10.7150/thno.45016 (2020).

20. Das, D., Santosh, K. C. \& Pal, U. Truncated inception net: COVID-19 outbreak screening using chest x-rays. Phys. Eng. Sci. Medicine 43, 915-925, DOI: 10.1007/s13246-020-00888-x (2020).

21. Zheng, C. et al. Deep learning-based detection for COVID-19 from chest CT using weak label. DOI: 10.1101/2020.03. 12.20027185 (2020).

22. Xu, X. et al. Deep learning system to screen coronavirus disease 2019 pneumonia. Eng. Vol. 6, Issue 10, Oct. 2020, Pages 1122-1129 DOI: 10.1016/j.eng.2020.04.010 (2020). 2002.09334. 
23. Barstugan, M., Ozkaya, U. \& Ozturk, S. Coronavirus (covid-19) classification using ct images by machine learning methods (2020). 2003.09424.

24. Chen, X., Yao, L. \& Zhang, Y. Residual attention u-net for automated multi-class segmentation of covid-19 chest ct images (2020). 2004.05645.

25. Li, L. et al. Using artificial intelligence to detect COVID-19 and community-acquired pneumonia based on pulmonary CT: Evaluation of the diagnostic accuracy. Radiology 296, E65-E71, DOI: 10.1148/radiol.2020200905 (2020).

26. Fan, D.-P. et al. Inf-net: Automatic covid-19 lung infection segmentation from ct images. IEEE Transactions on Med. Imaging 39, 2626-2637, DOI: 10.1109/TMI.2020.2996645 (2020).

27. Ratner, A. J., Ehrenberg, H. R., Hussain, Z., Dunnmon, J. \& Ré, C. Learning to compose domain-specific transformations for data augmentation (2017). 1709.01643.

28. Cubuk, E. D., Zoph, B., Mane, D., Vasudevan, V. \& Le, Q. V. Autoaugment: Learning augmentation policies from data (2019). 1805.09501.

29. Chen, Y. et al. Dual path networks (2017). 1707.01629.

30. He, K., Zhang, X., Ren, S. \& Sun, J. Deep residual learning for image recognition (2015). 1512.03385.

31. Huang, G., Liu, Z., van der Maaten, L. \& Weinberger, K. Q. Densely connected convolutional networks (2018). 1608. 06993.

32. Deng, J. et al. Imagenet: A large-scale hierarchical image database, DOI: 10.1109/cvprw.2009.5206848 (2009).

33. Hu, J., Shen, L., Albanie, S., Sun, G. \& Wu, E. Squeeze-and-excitation networks (2019). 1709.01507.

34. Zeiler, M. D. \& Fergus, R. Visualizing and understanding convolutional networks (2013). 1311.2901.

35. Doshi-Velez, F. \& Kim, B. Towards a rigorous science of interpretable machine learning (2017). 1702.08608.

36. Ribeiro, M. T., Singh, S. \& Guestrin, C. Nothing else matters: Model-agnostic explanations by identifying prediction invariance (2016). 1611.05817.

37. Cohen, J. P. et al. Covid-19 image data collection: Prospective predictions are the future (2020). 2006.11988.

38. Kermany, D. Large dataset of labeled optical coherence tomography (oct) and chest x-ray images, DOI: 10.17632/ RSCBJBR9SJ.3 (2018).

39. Khan, A. I., Shah, J. L. \& Bhat, M. M. Coronet: A deep neural network for detection and diagnosis of covid-19 from chest x-ray images. Comput. Methods Programs Biomed. 196, 105581, DOI: https://doi.org/10.1016/j.cmpb.2020.105581 (2020). 


\section{Figures}

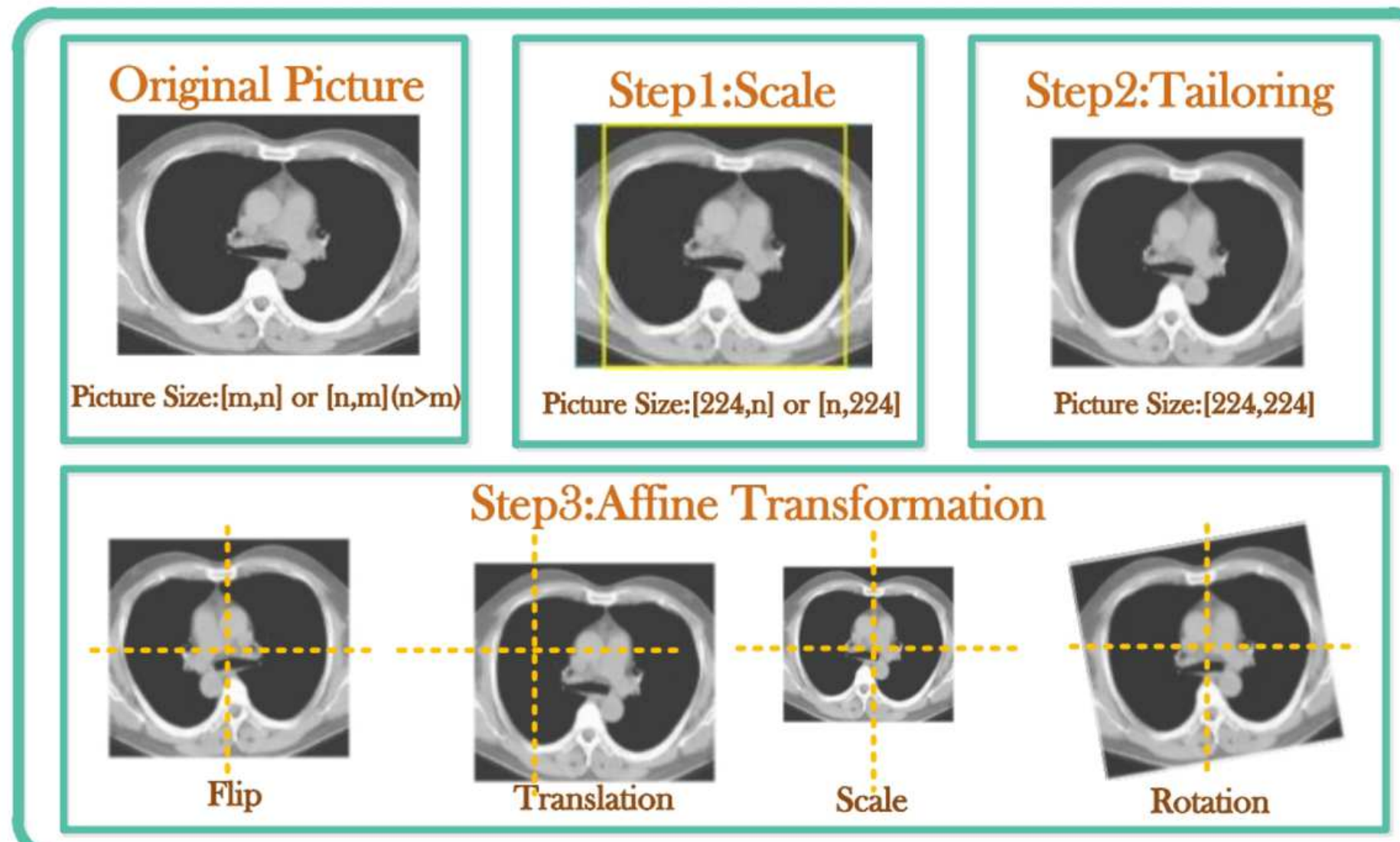

Figure 1

Overview of the Data Augmentation. Step1: Scale the narrow side of the image to size 224; Step2:

Randomly tailoring a picture of size $[224,224]$ from an image of $[224, n]$ or $[n, 224]$; Setp3: The $[224,224]$ size picture is processed by affine transformation: flip, rotate, scale. 


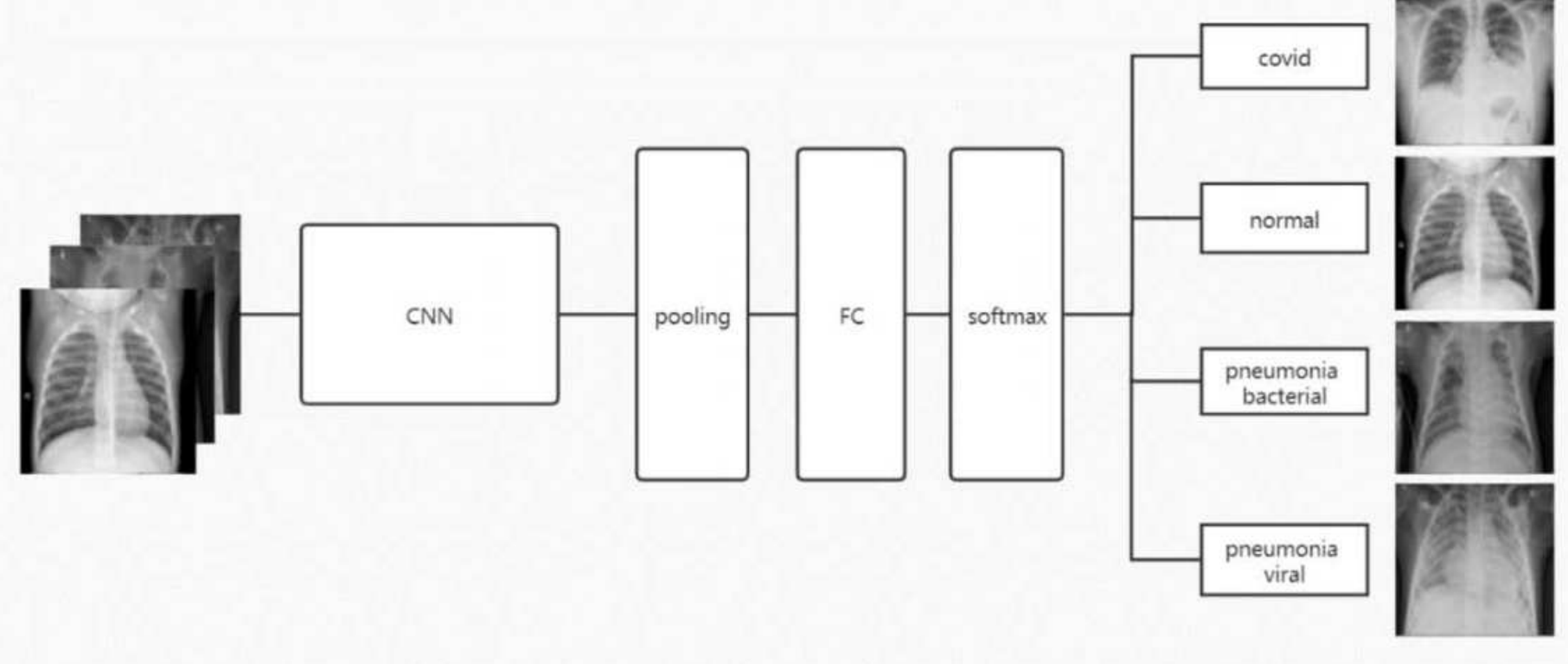

Figure 2

Network overall framework diagram
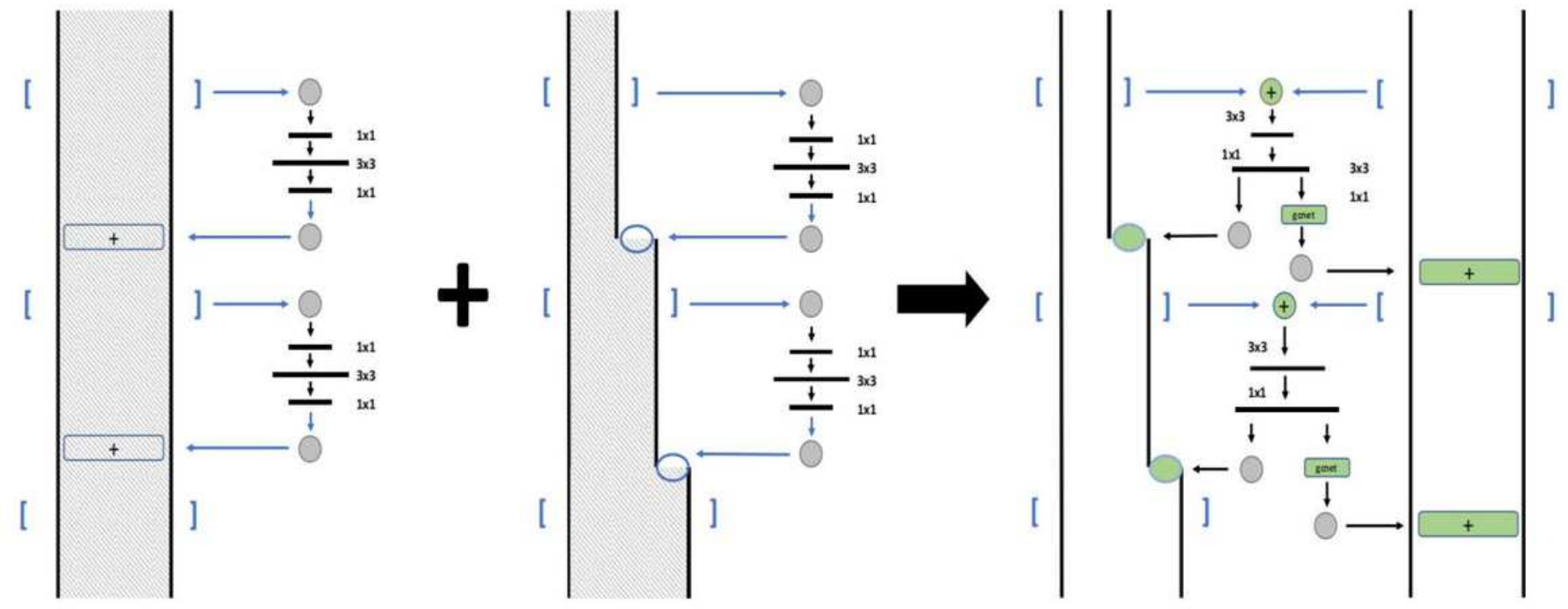

Figure 3

Network overall framework diagram 


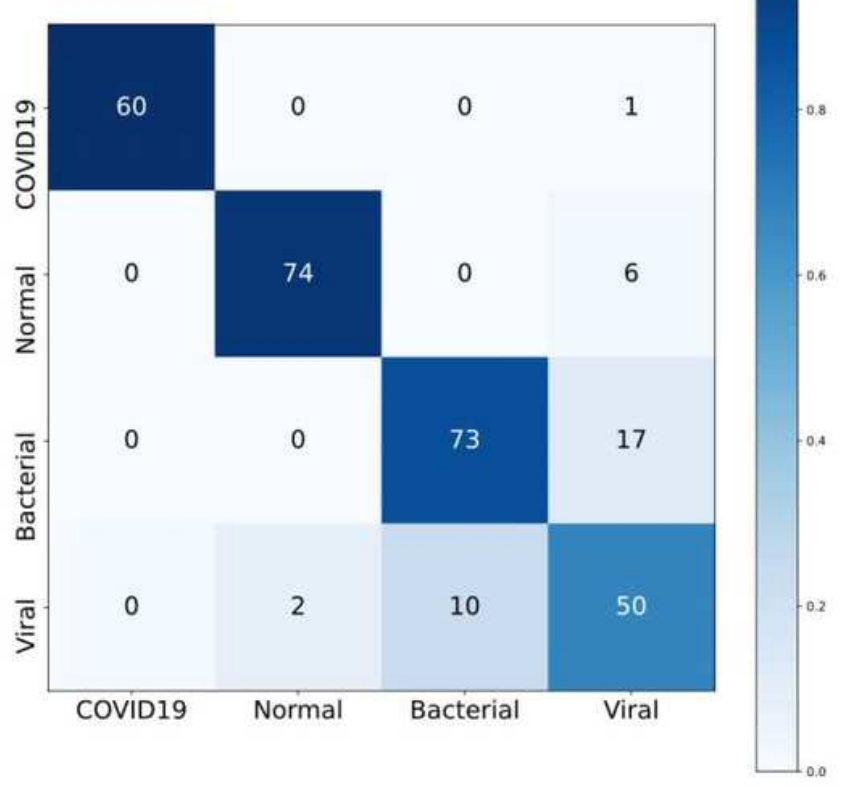

(a)

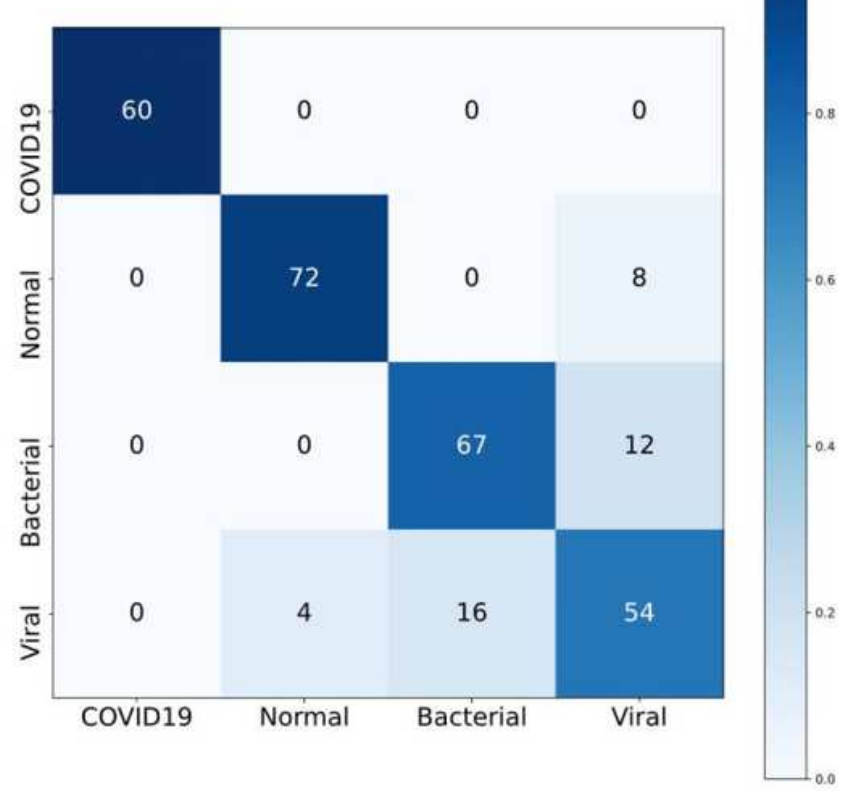

(b)

Figure 4

Schematic diagram of a four-level tripod-type atomic system driven by three coherent laser fields. 


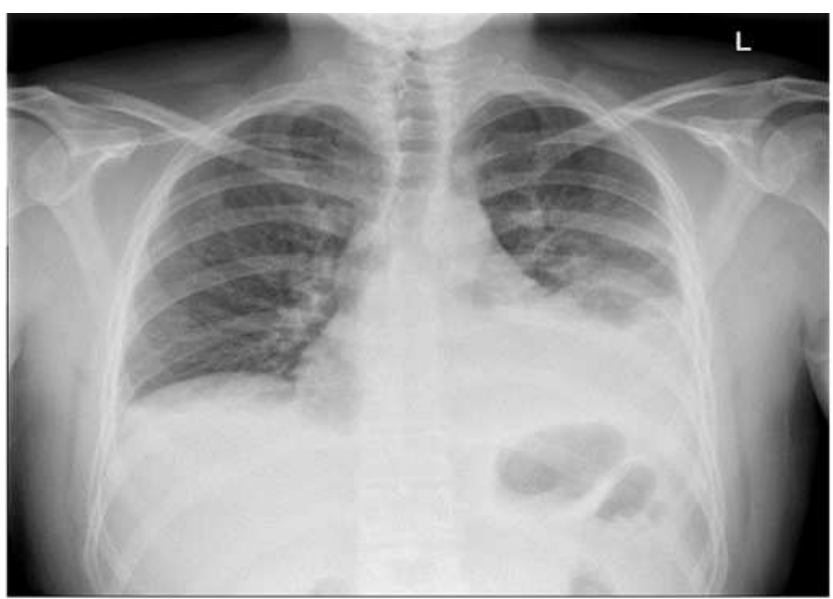

(a) Covid-19

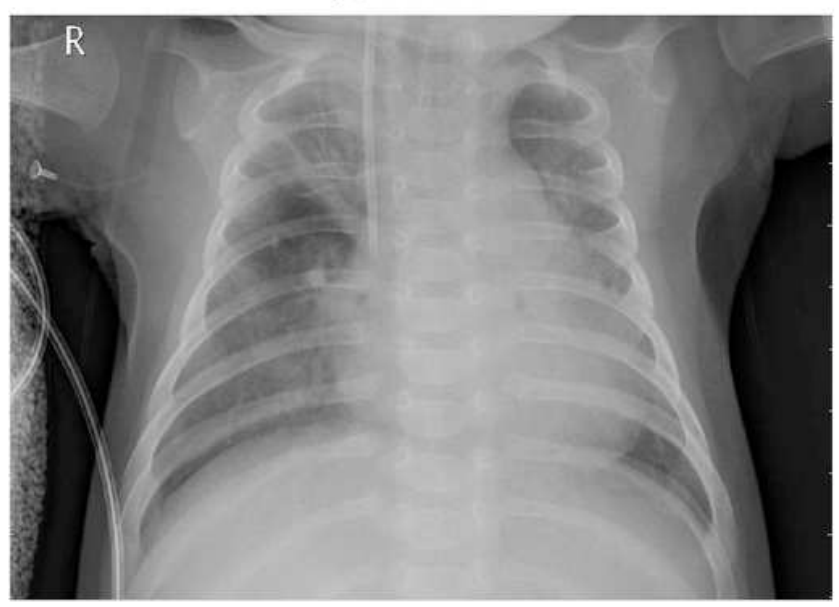

(c) Pneumonia Bacterial

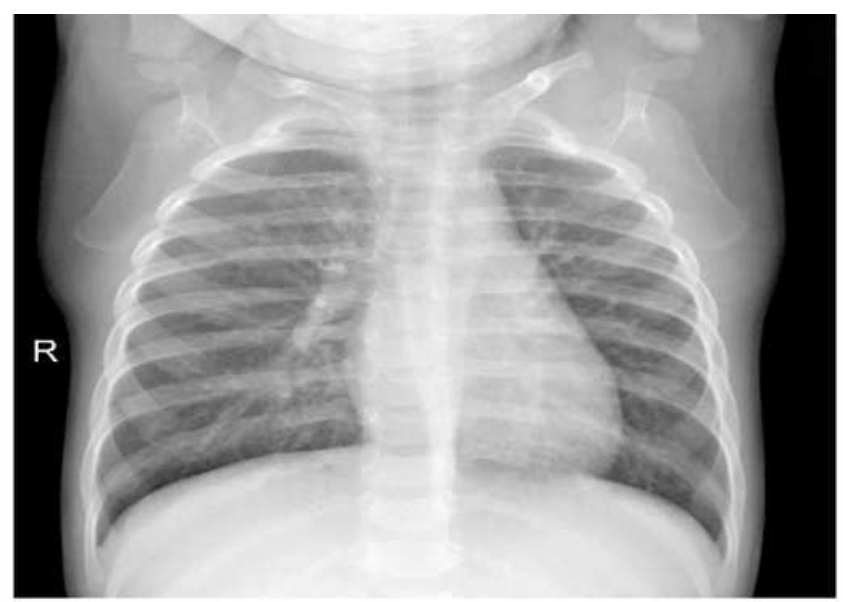

(b) Normal

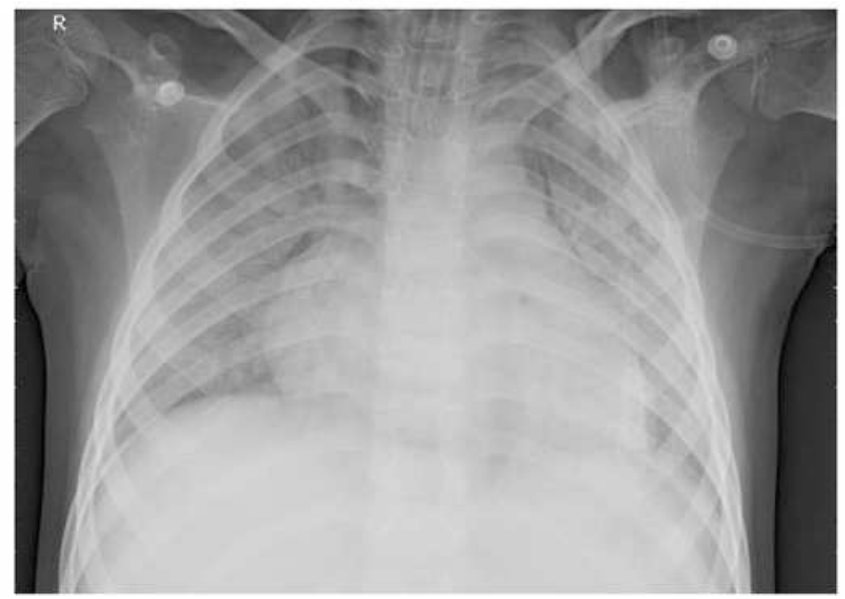

(d) Pneumonia Viral

Figure 5

Types of lung X-ray images 


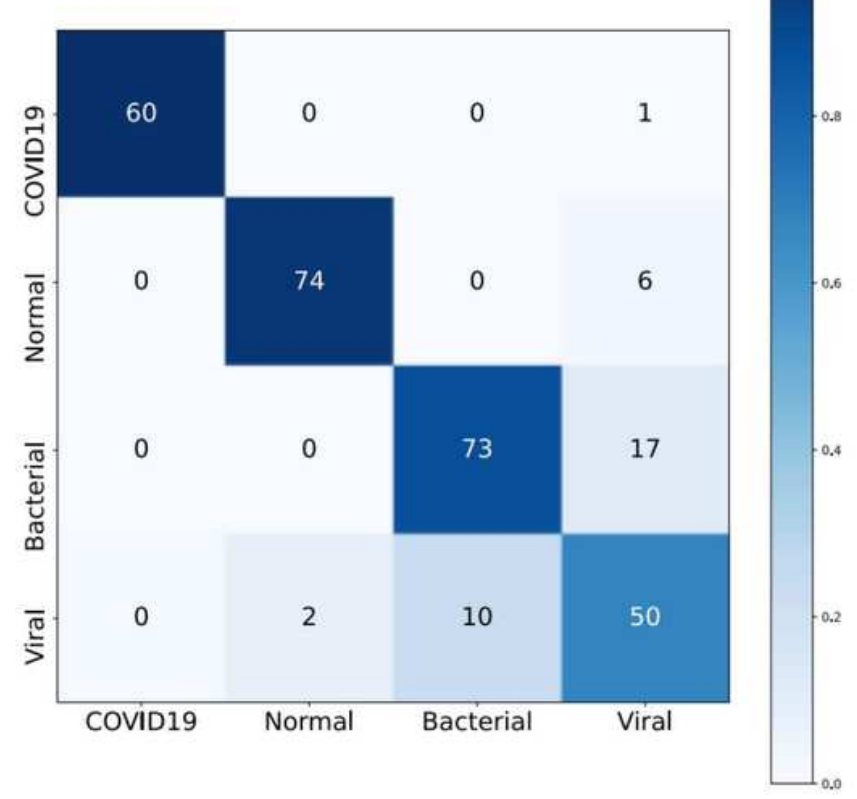

(a)

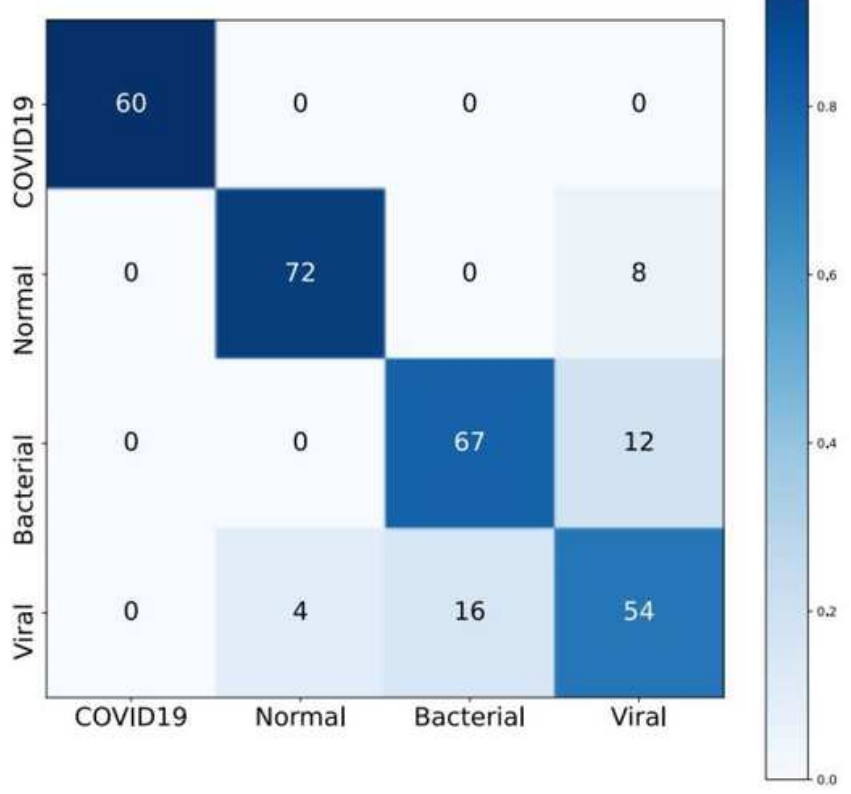

(b)

Figure 6

(a) the results of DPNNet network model training (b) the results of DPNN-SE network model training.

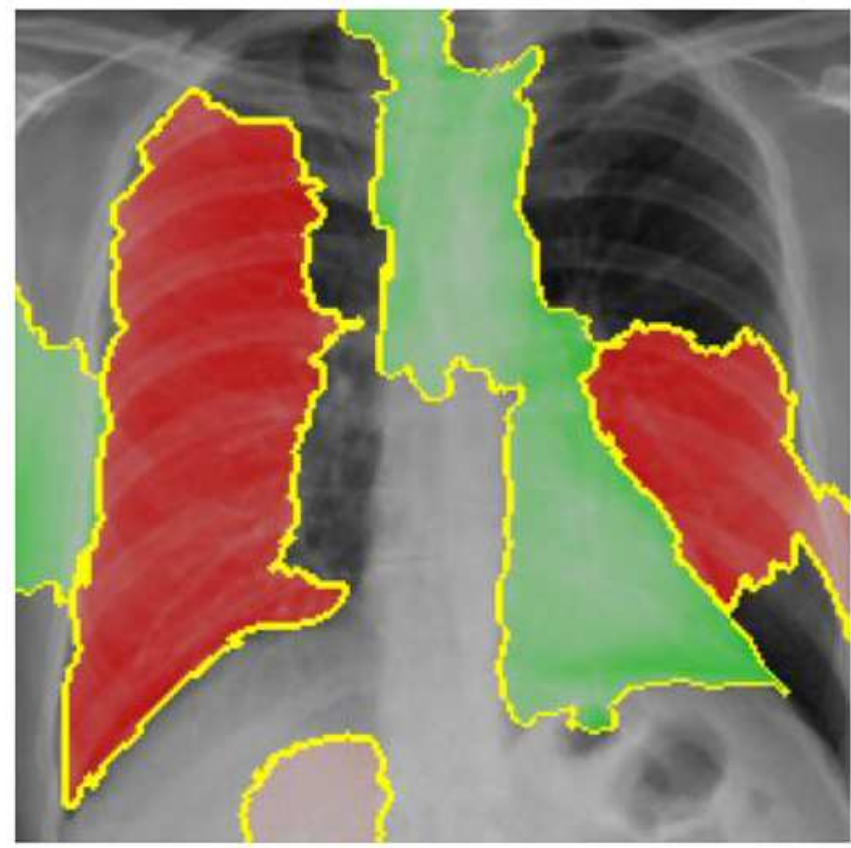

(a)

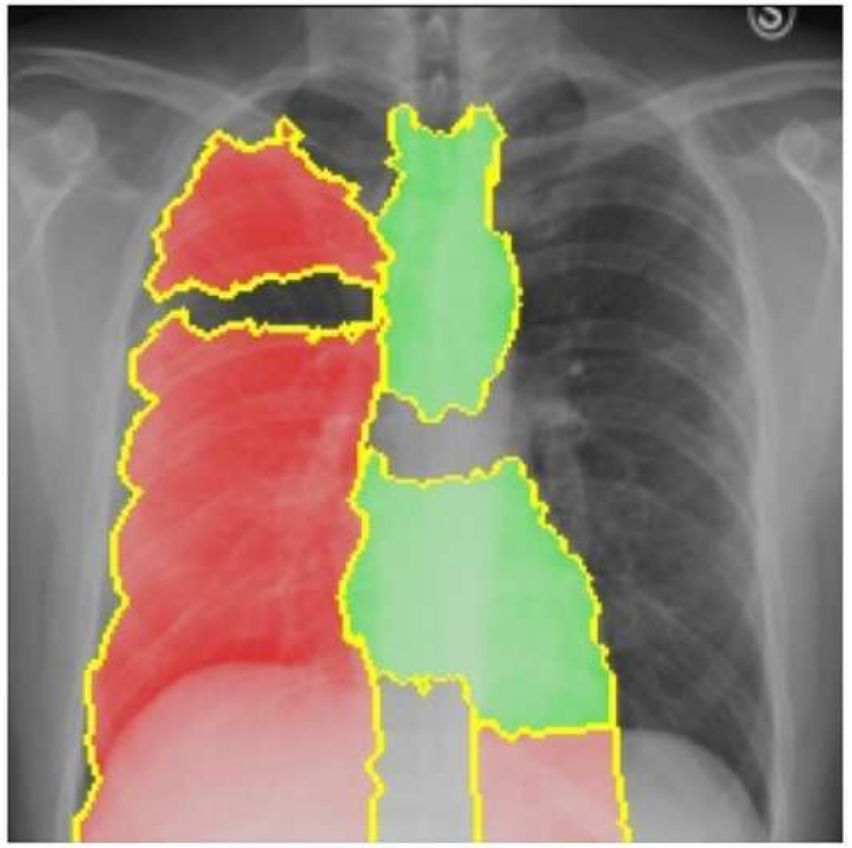

(b)

Figure 7

Examples of COVID-19 model activation maps. 